\title{
Unlocking the mysteries of the cumulus-oocyte complex-a critical cellular partnership for developmental competence
}

\author{
Jose Buratini ${ }^{1,2}$ (D) Pierre Comizzoli ${ }^{3}$ \\ Received: 10 February 2019 / Accepted: 12 February 2019 / Published online: 12 March 2019 \\ (C) Springer Science+Business Media, LLC, part of Springer Nature 2019
}

Structural and functional communications within the cumulus-oocyte complex (COC) are essential for gradual acquisition of oocyte developmental competence during folliculogenesis, and to ensure the occurrence of meiosis resumption after the LH peak, when the activation of the ovulatory cascade triggers final oocyte maturation in vivo. For many decades, cellular and molecular mechanisms during oocyte maturation have been explored in vitro in many species. This has led to the development of in vitro maturation (IVM), which has been intensively applied in combination with IVF in animal reproduction to enhance genetic gain speed in the last two decades. More recently, IVM has been also incorporated in strategies for human assisted reproduction [1]. This issue of JARG contains three interesting and elegant papers reinforcing the relevance of IVM in the ART context (Flageole et al.) (https://doi.org/10.1007/s10815-018-1394), the importance of the bovine model for IVM optimization (Razza et al.) (https://doi.org/10.1007/s10815-018-1365), and the predictive value of cumulus cell metabolism for oocyte developmental competence (Adrianensses et al.) (https://doi.org/10.1007/s10815-018-01398).

Although IVM has been mostly applied in patients with polycystic ovary syndrome (PCOS), it has been recognized as a valid alternative for ART protocols aiming to reduce the risk of ovarian hyperstimulation syndrome and treatment cost. However, the utilization of IVM has been largely limited by its still low efficiency. An interesting strategy to optimize IVM outcomes may be the development of new culture systems that

Jose Buratini

j.buratini@unesp.br

1 Biogenesi-Eugin, Reproductive Medicine Centre, Monza, Italy

2 Department of Physiology, Institute of Biosciences, Sao Paulo State University, Botucatu, Brazil

3 Smithsonian Conservation Biology Institute, National Zoological Park, Washington, DC, USA can mimick more closely the physiological environment where the cumulus-oocyte complex undergoes maturation. The case reported by Flageole and co-authors is an excellent illustration of the relevance of IVM in human reproductive medicine. It expands the applicability of IVM to patients with gonadotropin-resistant ovary syndromes, in which lack of gonadotropin responsiveness prevents the recovery of in vivo matured oocytes at the end of controlled ovarian stimulation. Moreover, by utilizing transfection experiments, the authors elegantly identify which of the two diagnosed FSHR mutations affects FSH signaling. There are numerous reports of FSHR activating and inactivating mutations in the literature causing hyperstimulation syndromes and ovarian failures, respectively. The report by Flageole and co-authors is the first to describe a successful fertility treatment including IVM for a patient with ovarian gonadotropin resistance.

Comparative studies in multiple animal species are key to make progress in human reproductive medicine [2]. Regarding COC biology, the bovine model has been highly informative and inspiring. A great deal of the recent advance in this field comes from studies in cattle, particularly those unrevealing the importance of cumulus-oocyte communication for oocyte maturation and developmental competence [3]. The paper by Razza and co-authors utilizes the bovine model to further explore the idea that holding meiosis progression at the beginning of IVM may allow a better synchronization of the cytoplasmic and nuclear oocyte compartments, thus favoring developmental competence. Interestingly, Razza et al. show that delaying meiosis with non-physiological cyclic nucleotide modulators before IVM may otherwise disturb subsequent embryo gene expression and development. The concept is not however deconstructed, and an interesting message from this study is that perhaps more physiological approaches are needed to delay meiosis and, more than that, prolong the time window for cumulus-oocyte exchange. As a matter of fact, the bovine model has also been important to demonstrate the importance of transzonal projections for the transfer of crucial small molecules and mRNA from cumulus cells to the oocyte [4]. And 
indeed, recent studies in cattle and humans evidence that the utilization of physiological players, such as natriuretic peptide $\mathrm{C}$, aiming to delay meiosis resumption and prolong cumulusoocyte communication before the activation of the ovulatory cascade, may be beneficial in IVM/IVF schemes $[5,6]$.

In the quest for non-invasive markers of oocyte quality, cumulus cells are excellent candidates.

In this issue of JARG, the importance of cumulus cell metabolism for oocyte developmental competence is favorably endorsed by the study of Adriaenssens et al., who demonstrate that embryo selection for fresh transfers can be markedly more accurate when specific patterns of cumulus gene expression are taken into consideration. Based on previous studies, the authors have selected cumulus expressed genes associated with oocyte quality that are involved in the regulation of cumulus expansion, ovulation, fertilization, and calcium metabolism (EFNB2, SASH1, and CAMK1D). Interestingly, this study also evidences that some cumulus expressed genes may present transcript levels proportional to oocyte developmental competence even after completion of maturation.

The COC still contains many secrets that will require a great effort to be gradually uncovered. The bovine model has been particularly valuable for a better understanding of the COC biology, which is absolutely essential for further development and application of human ART.

\section{References}

1. Coticchio G, Dal-Canto M, Guglielmo MC, Mignini-Renzini M, Fadini R. Human oocyte maturation in vitro. Int J Dev Biol. 2012;56(10-12):909-18. https://doi.org/10.1387/ijdb.120135gv.

2. Comizzoli P, Paulson EE, McGinnis LK. The mutual benefits of research in wild animal species and human-assisted reproduction. $\mathrm{J}$ Assist Reprod Genet. 2018;35(4):551-60. https://doi.org/10.1007/ s10815-018-1136-2.

3. Luciano AM, Sirard MA. Successful in vitro maturation of oocytes: a matter of follicular differentiation. Biol Reprod. 2018;98(2):162-9. https://doi.org/10.1093/biolre/iox149.

4. Macaulay AD, Gilbert I, Scantland S, Fournier E, Ashkar F, Bastien A, et al. Cumulus cell transcripts transit to the bovine oocyte in preparation for maturation. Biol Reprod. 2016;94(1):16. https://doi. org/10.1095/biolreprod.114.127571.

5. Soares ACS, Lodde V, Barros RG, Price CA, Luciano AM, Buratini J. Steroid hormones interact with natriuretic peptide $C$ to delay nuclear maturation, to maintain oocyte-cumulus communication and to improve the quality of in vitro-produced embryos in cattle. Reprod Fertil Dev. 2017;29(11):2217-24. https://doi.org/10.1071/RD16320.

6. Sanchez F, Lolicato F, Romero S, De Vos M, Van Ranst H, Verheyen $\mathrm{G}$, et al. An improved IVM method for cumulus-oocyte complexes from small follicles in polycystic ovary syndrome patients enhances oocyte competence and embryo yield. Hum Reprod. 2017;32(10): 2056-68. https://doi.org/10.1093/humrep/dex262.

Publisher's note Springer Nature remains neutral with regard to jurisdictional claims in published maps and institutional affiliations. 